\title{
Avaliação de Aspectos da Inteligência Emocional nas Técnicas de Pfister e Zulliger
}

\author{
Fabiano Koich Miguel ${ }^{1}$ \\ Universidade Estadual de Londrina, Londrina, PR, Brasil \\ Ana Carolina Zuanazzi \\ Anna Elisa de Villemor-Amaral \\ Universidade São Francisco, Itatiba, SP, Brasil
}

\begin{abstract}
Resumo
Inteligência emocional diz respeito à capacidade de perceber e compreender adequadamente as emoções e gerenciá-las de maneira adaptativa e construtiva. A regulação emocional faz parte do construto e é tradicionalmente avaliada por meio de histórias hipotéticas, o que se assemelha ao autorrelato. Embora desenvolvidas antes da proposta do construto inteligência emocional, as técnicas projetivas, consideradas instrumentos de desempenho, também apresentam indicadores referentes à regulação das emoções. A presente pesquisa teve como objetivo estudar quais variáveis do Teste das Pirâmides Coloridas de Pfister estariam associadas a um indicador de regulação cognitiva das emoções no Teste das Manchas de Tinta de Zulliger. Participaram 98 pessoas, sendo 57,1\% do sexo feminino. Análise de regressão linear identificou que as frequências de Tapetes Puros e de Tapetes Furados (ambas negativamente) do Pfister foram preditoras da fórmula de regulação emocional do Zulliger. Considera-se que aspectos de inteligência emocional podem ser encontrados também em técnicas projetivas. A associação entre as variáveis do Pfister e do Zulliger são discutidos quanto à sua indicação de saúde psicológica.
\end{abstract}

Palavras-chave: Inteligência emocional, técnicas projetivas, testes de autoexpressão, avaliação psicológica.

\section{Assessment of Emotional Intelligence Aspects in the Methods of Pfister's and Zulliger's}

\begin{abstract}
Emotional intelligence relates to the ability to adequately perceive and understand emotions and to manage them in an adaptive and constructive manner. Emotion regulation is part of the construct and is traditionally assessed by way of hypothetical stories, which are similar to self-reports. Although developed prior to the proposal of the emotional intelligence construct, projective techniques, which are considered performance instruments, also feature indicators of emotion regulation. The present study's aim was to discover which variables in Pfister's Color Pyramid Test would be associated with an indicator of cognitive emotion regulation in the Zulliger Inkblot Test. Ninety-eight people participated in the survey, $57.1 \%$ of which were women. Linear regression analysis showed that the frequencies of Pure Carpet
\end{abstract}

Endereço para correspondência: Universidade Estadual de Londrina, Departamento de Psicologia e Psicanálise, Centro de Ciências Biológicas, Campus Universitário, Caixa Postal 10011, Londrina, PR, Brasil 86057-970. Fone: (43) 3371-4397. E-mail: fabiano@avalpsi.com.br

Os autores agradecem a colaboração das turmas 58, 59 e 60 de Psicologia da Universidade Estadual de Londrina na aplicação dos testes psicológicos. 
and Bored Carpet (both negative) in the Pfister test were predictors of the Zulliger emotion-regulation formula. It is believed that aspects of emotional intelligence can also be found in projective techniques. The association between Pfister's and Zulliger's variables is discussed as an indicator of psychological health.

Keywords: Emotional intelligence, projective techniques, self-report inventories, psychological assessment.

\section{Evaluación de Aspectos de la Inteligencia Emocional en las Técnicas de Pfister y Zulliger}

\section{Resumen}

La inteligencia emocional es la capacidad de percibir y comprender adecuadamente las emociones y gestionarlos de una manera adaptativa y constructiva. La regulación emocional es parte del constructo y se evalúa tradicionalmente por historias hipotéticas, que es similar a la auto-informe. Aunque se desarrolló antes de la propuesta de la construcción de la inteligencia emocional, las técnicas proyectivas, instrumentos de desempeño, también disponen de indicadores de regulación emocional. Esta investigación tuvo como objetivo estudiar qué variables de la Prueba de Pirámides Coloridas del Pfister estarían asociadas con un indicador de regulación cognitiva de las emociones de la Prueba de Manchas de Tinta de Zulliger. Participaron 98 personas, con 57,1\% mujeres. El análisis de regresión lineal mostró que la frecuencia de Tapetes Puros y Tapetes Perforados (ambos negativamente) en el Pfister fueron predictores de la fórmula de regulación emocional en Zulliger. Consideramos que aspectos de la inteligencia emocional también se pueden encontrar en las técnicas proyectivas. Se discute la asociación entre variables Pfister y Zulliger como indicadores de la salud psicológica.

Palabras clave: Inteligencia emocional, técnicas proyectivas, pruebas de auto-expresión, evaluación psicológica.

Inteligência emocional diz respeito à capacidade de identificar as expressões emocionais, tanto de si mesmo quanto dos outros, compreender as situações desencadeadoras e as possíveis consequências, e utilizar essas informações de maneira a promover bem-estar e crescimento social (Caruso, Salovey, Brackett, \& Mayer, 2015; Mayer, Roberts, \& Barsade, 2008). O construto costuma ser composto por quatro áreas: percepção emocional, referente à capacidade de reconhecer adequadamente as expressões; facilitação do pensamento, que remete à capacidade de utilizar as emoções para melhor desempenhar uma tarefa; compreensão emocional, relacionada com a capacidade de perceber como as emoções transitam e se mesclam ao longo do tempo; e gerenciamento emocional, que se refere à capacidade de se engajar em pensamentos e ações que permitam a resolução de conflitos e favoreçam a adaptação (Mayer \& Salovey, 1999).
Existem modelos que compreendem a inteligência emocional como um traço, que pode ser avaliado por meio de inventários de autorrelato, apresentando correlação com outros traços de personalidade (Petrides, Pita, \& Kokkinaki, 2007; Woyciekoski \& Hutz, 2010). Por outro lado, existem os modelos que tratam a inteligência emocional como capacidade cognitiva, sendo avaliada por meio de testes de desempenho e apresentando correlações com outros tipos de raciocínio (Caruso et al., 2015; Miguel, 2010; Zeidner, Shani-Zinovich, Matthews, \& Roberts, 2005). Os testes de desempenho intelectual ou desempenho máximo costumam ser recomendados ao se avaliar uma capacidade cognitiva por não dependerem de adequada autopercepção do sujeito em relação a suas habilidades, como acontece no caso do autorrelato, mas sim colocam à prova a utilização da habilidade. 
Contudo, embora as tarefas das áreas de percepção, facilitação e compreensão possam ser operacionalizadas em instrumentos por meio de itens com respostas certas e erradas (o que colocaria à prova a capacidade do avaliando), a área de gerenciamento seria mais difícil de operacionalizar em um teste. O formato tradicional, nesses casos, tem sido apresentar histórias e perguntar ao avaliando o que o personagem deve fazer para melhor resolver a situação (Bueno, 2008; Mayer, Salovey, \& Caruso, 2002). Esse formato, portanto, avalia o conhecimento que a pessoa tem sobre como resolver um conflito, não necessariamente significando que coloque aquele conhecimento em prática ao se deparar com situações que exijam regulação emocional. Nesse sentido, o formato se assemelharia ao autorrelato, no que diz respeito ao tipo de resultado e à sua interpretação.

Por outro lado, a avaliação do gerenciamento emocional que não fosse por autorrelato é algo difícil de ser feita pelo psicólogo, pois envolveria acompanhar o indivíduo em diversos momentos de sua vida, no caso da observação direta, ou então criar uma situação em que se exigisse tal capacidade, como as dinâmicas de grupo, por exemplo. Outra possibilidade seria propor tarefas de desempenho que avaliassem o gerenciamento indiretamente. Por exemplo, Bueno (2013) desenvolveu um teste de regulação emocional semelhante à tarefa de Stroop, em que o avaliando deve ler palavras e nomear a cor em que ela foi escrita, enquanto imagens de valor afetivo positivo e negativo são apresentadas ao fundo como distratores emocionais da tarefa. Embora a estrutura fatorial do teste tenha se mostrado adequada, os resultados ainda não corresponderam à expectativa de desempenho, sendo necessários mais estudos.

Considerando-se que a capacidade de gerenciamento das emoções também é estudada fora da área de inteligência emocional, pode-se argumentar que outros instrumentos seriam capazes de avaliá-la indiretamente. É possível encontrar indicadores nesse sentido nos testes projetivos - também chamados de técnicas de autoexpressão ou de avaliação da personalidade por desempenho (Meyer \& Kurtz, 2006; Miguel, 2014; Villemor-Amaral, 2008).

Por exemplo, as técnicas de manchas de tinta de Rorschach e Zulliger incluem a fórmula FC-CF-C (dependendo do sistema de interpretação utilizado, também é representada como $\mathrm{FC}: \mathrm{CF}+\mathrm{C}$ ou $(\mathrm{CF}+\mathrm{C}) / \mathrm{SumC})$. Essa fórmula indica a proporção de determinantes de cor com forma predominante em relação aos determinantes de cor em que a forma não é predominante ou está ausente. As pesquisas têm demonstrado que a maior proporção de respostas determinadas pela cor, cuja forma é clara e precisa (representada na fórmula FC-CF-C com valores positivos), está associada a maior modulação da expressão emocional e filtro cognitivo, implicando em regulação dos afetos e menor impulsividade ou reatividade frente às situações (Berant, Mikulincer, Shaver, \& Segal, 2005; Exner \& Sendín, 1999; Malone et al., 2013; Meyer, Viglione, Mihura, Erard, \& Erdberg, 2011; Villemor-Amaral \& Primi, 2012). Trata-se, portanto, de um indicador de maturidade na regulação das emoções, havendo domínio cognitivo da situação.

Embora Herman Rorschach tenha sido o primeiro a chamar a atenção para a proporção entre os diferentes tipos de repostas determinadas pela cor como sendo um indicador de controle e maturidade emocional, a relação entre as reações à cor como correlatas às reações emocionais já era um conhecimento muito antigo, compartilhado culturalmente. Mas foi somente com o avanço nas neurociências que essa relação pode ser demonstrada cientificamente, quando estudos verificaram a predominância das mesmas áreas de especialização cerebral para os diferentes modos de processar a cor e a emoção (Levy \& Trevarthen, 1981). Mais tarde, em sua revisão sobre os estudos que associavam cor à emoção, Bash (1984) conclui que em realidade a percepção da cor é puramente sensorial em contraposição ao reconhecimento de forma, estrutura e qualidades dos objetos, que envolvem mais as funções cognitivas - o mesmo ocorrendo com as emoções.

Outra técnica projetiva que possui indicadores relacionados ao gerenciamento emocional é o teste das Pirâmides Coloridas de Pfister (Vil- 
lemor-Amaral, 2012). A frequência de algumas cores e a preferência por certos aspectos formais ao se construir as pirâmides utilizando-se de quadrículos coloridos podem indicar o nível de capacidade de regular os estados afetivos. No Pfister, essa capacidade é demonstrada quanto melhor for a organização da forma final da pirâmide, ou, inversamente, se a desorganização da forma é muito grande, supõe-se fraco gerenciamento das emoções.

Considerando a capacidade dessas duas técnicas de avaliar o modo de lidar com os afetos e, portanto, gerenciamento emocional, partiu-se da hipótese de que bom aspecto formal no Pfister estaria correlacionado com uma fórmula FC-CF-C positiva e desorganização acentuada no aspecto formal das pirâmides se associaria à fórmula FC-CF-C negativa. Com base nessas hipóteses, a presente pesquisa teve por objetivo estudar quais aspectos formais do Pfister estariam relacionados com a regulação emocional de acordo com a fórmula FC-CF-C do Zulliger.

\section{Método}

\section{Participantes}

Participaram da pesquisa 98 pessoas, sendo $56(57,1 \%)$ do sexo feminino, moradores da cidade de Londrina-PR e região. A idade média foi de 21,25 $(D P=3,34)$, com mínimo de 18 e máximo de 35 anos. Todos os participantes eram estudantes de graduação (de diversos cursos como Psicologia, Direito, Biologia, entre outros) ou haviam concluído o ensino médio.

\section{Instrumentos}

\section{Teste de Zulliger Sistema Compreensivo}

O teste foi desenvolvido por Hans Zulliger na década de 1940 tendo como base o teste de Rorschach. O Sistema Compreensivo foi desenvolvido por Exner como uma proposta de avanço para ao Rorschach e, dado os benefícios alcançados, esse sistema foi também adaptado para o Zulliger, com pesquisas de normatização e validação para a população brasileira realizadas por Villemor-Amaral e Primi (2012). O teste consis- te na interpretação de manchas de tinta. O material é constituído por três cartões contendo manchas de tinta, uma preta e branca, uma colorida e uma contendo preto, branco e vermelho. Essas são mostradas uma de cada vez, solicitando-se à pessoa que diga com que elas se parecem. As respostas obtidas são codificadas com base em um criterioso sistema de classificação que leva em conta diversos aspectos contidos no conceito visto e na verbalização, dentre eles a área onde a pessoa localiza sua resposta; que aspectos da mancha sugeriram a ideia; a adequação aos contornos da mancha do objeto percebido; tipo de conteúdo e assim por diante. Interpreta-se o resultado de fórmulas e cálculos feitos com base na frequência dessas categorias, o que torna o teste complexo do ponto de vista psicométrico. A combinação dos resultados, agrupados de modos diversos, traz informações sobre o funcionamento cognitivo, dinâmica afetiva, autopercepção, capacidade de relacionamento, capacidade de controle e tolerância ao estresse como também alguns indicadores psicopatológicos. Como já mencionado, nas respostas determinadas pela cor da mancha, o grau de predominância da forma na definição do conceito percebido é um indicador de capacidade de controle na expressão dos afetos.

\section{Teste das Pirâmides Coloridas de Pfister}

O teste foi criado na década de 1950 por Max Pfister. Consiste em solicitar ao examinando que preencha o esquema de três pirâmides com quadrículos coloridos. As pirâmides são feitas uma de cada vez e o examinado dispõe de um jogo composto por 50 quadrículos de cada uma das 24 tonalidades que se reduzem a um total de 10 cores: verde, azul, vermelho, amarelo, laranja, violeta, marrom, preto, branco e cinza. Para a análise dos resultados leva-se em conta não somente a frequência das cores utilizadas, mas sobretudo o aspecto formal que configura cada pirâmide, o modo da pessoa executar a tarefa, a maior ou menor constância com que utiliza as cores ao longo das três pirâmides, principais combinações, entre outros indicadores. São de especial importância as considerações a respeito do grau de organização das cores sobre o esque- 
ma da pirâmide, que resulta em aspectos formais mais ou menos estruturados. As informações centrais obtidas com os resultados permitem inferir principalmente sobre o nível de maturidade emocional nas interações sociais, sendo possível eventualmente levantar hipóteses sobre a capacidade intelectual da pessoa, dada a predominância de mecanismos cognitivos ao lidar com as cores, dispondo-as de modo harmonioso e organizado ao executar a tarefa.

\section{Procedimentos de Coleta de Dados}

As aplicações dos testes ocorreram dentro da disciplina Teorias e Técnicas Projetivas, componente do curso de graduação de Psicologia da Universidade Estadual de Londrina, ao longo de um período de dois anos. Os estudantes foram treinados pelo primeiro autor desta pesquisa na aplicação dos instrumentos, sendo a aplicação em dupla e supervisionada pelo docente. Os estudantes convidaram os participantes para a avaliação, que foi realizada na clínica psicológica da mesma universidade em um único encontro.

Todos os participantes receberam inicialmente o Termo de Consentimento Livre e Esclarecido de atendimento na clínica-escola. Após a leitura e concordando com a participação, iniciou-se a aplicação. Todos os procedimentos éticos foram respeitados.

$\mathrm{O}$ teste de Zulliger foi aplicado primeiro, seguido do teste de Pfister. As aplicações seguiram a padronização dos instrumentos de acordo com seus respectivos manuais. Os protocolos que não seguiram uma aplicação adequada (por exemplo, instruções incompletas) foram excluídos da pesquisa.

\section{Procedimentos de Análise de Dados}

Originalmente foram coletados 180 protocolos. Após se excluir protocolos com aplicações inadequadas, selecionou-se somente aqueles que continham respostas de cor. Para tal, levou-se em conta as recomendações feitas pelo novo sistema de interpretação do Rorschach (Rorschach Performance Assessment System, ou R-PAS) que sugere a utilização de pelo menos quatro respostas de cor para o cálculo da fórmula de predominância da forma, para evitar proporções pouco precisas (Meyer et al., 2011). Porém, essa quantidade é difícil de se obter no Zulliger, pois a frequência de respostas neste teste tende a ser bem menor do que no Rorschach. Assim, apenas 19 participantes produziram quatro ou mais respostas de cor, e 42 produziram três ou mais. Para permitir maior variabilidade de escores, optou-se pelo critério de produção de duas ou mais respostas de cor, resultando nos 98 participantes da pesquisa.

A variável independente do Zulliger foi calculada subtraindo de FC os valores de $\mathrm{CF}$ e $\mathrm{C}$, e então dividindo pelo total de respostas no teste, R. A divisão pelo número de respostas é sugerida em pesquisas para evitar resultados enviesados devido à variabilidade de $\mathrm{R}$ (Meyer et al., 2011). Foi feita análise de regressão linear pelo método enter, buscando-se predizer o escore (FC-CF-C)/R do Zulliger por meio de variáveis numéricas do Pfister.

Foram selecionados os aspectos formais do Pfister que, de acordo com seu manual (Villemor-Amaral, 2012), estavam especificamente relacionadas ao controle ou descontrole emocional devido à adequada utilização ou à menor participação do pensamento, a saber: Tapete Puro, Tapete Furado, Tapete com Início de Ordem, Formações em Camadas e Estrutura Simétrica. No caso das Formações em Camadas, os três subtipos (monotonais, monocromáticas e multicromáticas) compartilham a mesma interpretação no sentido de nível pouco amadurecido de regulação das emoções, por isso foi considerada a frequência total desse aspecto.

O teste de Pfister também possui as frequências de cores como variáveis numéricas, porém elas não foram utilizadas neste estudo pois sua interpretação não é linear. Isso quer dizer que valores extremos que indicam excesso de utilização da cor, ou sua redução no teste, podem refletir aspectos negativos. Por exemplo, a cor Verde é considerada um indicador de capacidade de insight e empatia, desde que se apresente dentro dos níveis médios, o que denota boa qualidade nos relacionamentos interpessoais. Embora o nível reduzido dessa cor indique uma diminuição 
nessas habilidades, o nível aumentado do Verde não teria uma interpretação positiva, mas sim indicaria excesso de estimulação que sobrecarregaria a pessoa e conduziria a um desequilíbrio emocional.

Para análise do efeito estatístico, levou-se em consideração a seguinte referência: magnitudes abaixo de 0,20 como fracas; entre 0,20 e 0,40 como moderadas; acima de 0,40 como fortes (Cohen, 1992; Hemphill, 2003).

Tabela 1

Estatística Descritiva das Variáveis Estudadas

\begin{tabular}{ccccc}
\hline Variável & Média & $D P$ & Mínimo & Máximo \\
\hline (FC-CF-C)/R & $-0,01$ & 0,25 & $-0,67$ & 0,50 \\
Tapete puro & 0,10 & 0,31 & 0 & 1 \\
Tapete furado & 0,07 & 0,33 & 0 & 2 \\
Tapete com início de ordem & 0,28 & 0,55 & 0 & 3 \\
Formação em camadas (total) & 0,94 & 1,04 & 0 & 3 \\
Estrutura simétrica & 0,19 & 0,46 & 0 & 2 \\
\hline
\end{tabular}

A fórmula gerada por meio da análise de regressão linear encontra-se na Tabela 2 . O resultado apontou dois aspectos formais do Pfister como preditores do desempenho no Zulliger no que diz respeito à regulação cognitiva das emoções, que foram as quantidades de Tapetes $\mathrm{Pu}-$ ros e de Tapetes Furados, ambos negativamente. O índice $R^{2}$ desse modelo foi 0,15 . Em seguida foi executado modelo de regressão linear apenas com as duas variáveis, que também se encontra na Tabela 2. O $R^{2}$ foi de 0,12 .

A fim de verificar colinearidade, as variáveis utilizadas na regressão foram correlacionadas entre si. Os resultados são apresentados na parte inferior da Tabela 2. Foi encontrada uma correlação significativa entre a quantidade de Tapetes com Início de Ordem e a quantidade total de Formação em Camadas, embora a magnitude dessa correlação não tenha se mostrado elevada. De fato, ao se executar a regressão linear novamente, dessa vez excluindo a variável Formação em Camadas, não houve alteração expressiva dos resultados.

\section{Resultados}

As estatísticas descritivas das variáveis estudadas estão apresentadas na Tabela 1. Pode-se perceber que a maior frequência foi do aspecto Formação em Camadas, enquanto os tapetes e a estrutura tiveram frequência menor. Essas distribuições se assemelharam bastante àquelas relatadas nas pesquisas de normatização do Pfister (Villemor-Amaral, 2012). 
Tabela 2

Regressão Linear com Variáveis do Pfister Predizendo (FC-CF-C)/R no Zulliger e Correlações entre as Variáveis

\begin{tabular}{|c|c|c|c|c|c|}
\hline $\begin{array}{c}\text { Regressões } \\
\text { Modelos e Variáveis }\end{array}$ & Beta & $B$ & IC $(95 \%)$ & $t$ & $p$ \\
\hline \multicolumn{6}{|l|}{ Modelo 1 (todas variáveis) } \\
\hline Constante & & 0,02 & $-0,07$ a 0,11 & 0,48 & 0,631 \\
\hline Tapete puro & $-0,26$ & $-0,22$ & $-0,38$ a $-0,06$ & $-2,66$ & 0,009 \\
\hline Tapete furado & $-0,23$ & $-0,17$ & $-0,32$ a $-0,02$ & $-2,31$ & 0,023 \\
\hline Tapete com início de ordem & $-0,04$ & $-0,02$ & $-0,11$ a 0,07 & $-0,40$ & 0,689 \\
\hline Formação em camadas (total) & 0,07 & 0,02 & $-0,03$ a 0,07 & 0,70 & 0,489 \\
\hline Estrutura simétrica & $-0,12$ & $-0,07$ & $-0,18$ a 0,04 & $-1,20$ & 0,235 \\
\hline \multicolumn{6}{|l|}{ Modelo 2 (final) } \\
\hline Constante & & 0,02 & $-0,03$ a 0,07 & 0,79 & 0,430 \\
\hline Tapete puro & $-0,26$ & $-0,22$ & $-0,38$ a $-0,06$ & $-2,69$ & 0,008 \\
\hline \multirow[t]{3}{*}{ Tapete furado } & $-0,22$ & $-0,17$ & $-0,32$ a $-0,03$ & $-2,32$ & 0,023 \\
\hline & & & & & \\
\hline & 1 & 2 & 3 & 4 & 5 \\
\hline \multicolumn{6}{|l|}{ 1. Tapete puro } \\
\hline 2. Tapete furado & 0,03 & & & & \\
\hline 3. Tapete com início de ordem & 0,07 & 0,00 & & & \\
\hline 4. Formação em camadas (total) & $-0,14$ & $-0,11$ & $-0,28 * *$ & & \\
\hline 5. Estrutura simétrica & $-0,14$ & $-0,09$ & $-0,08$ & $-0,19$ & \\
\hline
\end{tabular}

$* * p<0,01$.

Tapete Furado como preditor de maior (FC-CF-C)/R no Zulliger. Segundo o manual do Pfister (Villemor-Amaral, 2012), Tapete Furado indica dissociações ou perturbações no pensamento e costumam ser produzidos em maior quantidade por pacientes esquizofrênicos, embora não exclusivamente. Tais pacientes tipicamente exibem déficits na utilização de estratégias cognitivas para o gerenciamento das emoções (O'Driscoll, Laing, \& Mason, 2014; Strauss et al., 2013), o que indica coerência para os resultados encontrados, inclusive a associação com Tapete Puro.

Deve-se levar em conta que a magnitude dos efeitos foi moderada a baixa $(-0,26$ para Tapetes Puros e - 0,23 para Tapetes Furados), o que indica que os construtos avaliados pelas variáveis do
Pfister apresentam leve relação com a fórmula do Zulliger, mas não o suficiente para se afirmar que tratam exatamente da mesma coisa. Para afirmar isso, uma maior magnitude de efeito seria necessária. Portanto, não é possível afirmar que as ocorrências de Tapete Puro e Tapete Furado estejam medindo regulação emocional da mesma maneira que a fórmula (FC-CF-C)/R do Zulliger. De fato, o valor de $R^{2}$ da regressão linear do modelo final indica apenas $12 \%$ de variância compartilhada entre Zulliger e os aspectos do Pfister, reforçando que o poder explicativo do resultado de um teste pelo outro é relativamente baixo e, portanto, não se deve entender os dois como avaliando o mesmo construto. Contudo, a relação significativa daqueles dois aspectos 
formais sugere que essas três variáveis estejam se referindo a aspectos relacionados de um processo psicológico. Assim, é possível que as variáveis do teste estejam compartilhando parte do mesmo construto - regulação emocional.

Ademais, os outros aspectos formais que faziam parte das hipóteses (Tapete com Início de Ordem, Formação em Camadas, Estrutura Simétrica) não apresentaram relação significativa com a fórmula do Zulliger, demonstrando efeitos leves ou desprezíveis. Esse resultado não exclui a possibilidade de que esses aspectos formais estejam avaliando capacidade de regulação das emoções, porém o processo psicológico utilizado nesses aspectos mostrou-se pouco ou nada relacionado com o controle cognitivo segundo o Zulliger.

Levando-se em conta os resultados apresentados, observa-se coerência na associação entre as variáveis, no sentido de que menor frequência de pirâmides de aspecto formal Tapete Puro e Tapete Furado estão levemente associadas à fórmula (FC-CF-C)/R equilibrada no Zulliger, indicadora de maior controle cognitivo sobre as emoções. Dessa maneira, é possível verificar que esse aspecto da inteligência emocional em particular - a regulação cognitiva das emoções - pode estar presente nas duas técnicas estudadas, havendo correlação entre os indicadores de ambas, embora, como visto, as duas técnicas estejam avaliando o construto de maneiras pouco semelhantes, embora relacionadas. Portanto, considera-se que esses instrumentos podem contribuir para a avaliação também sob essa perspectiva, mesmo que eles não tenham sido originalmente desenvolvidos para avaliar especificamente o construto de inteligência emocional, que foi definido posteriormente à criação de ambas as técnicas. Isso ocorre naturalmente, uma vez que a utilização das funções cognitivas na regulação dos afetos já vem sendo estudada em avaliação psicológica como regulação emocional, mesmo anteriormente à proposta da inteligência emocional.

Como discutido, deve-se considerar essas associações baixas a moderadas como hipóteses de interpretação ao se utilizar esses instrumentos. Além disso, não se pode perder de vista o contexto de cada protocolo em sua totalidade. Isso porque os excessos podem denotar desequilíbrio e não uma maior habilidade. No caso do FC, a dificuldade de adaptação emocional se daria pelo excesso de racionalidade que impediria a expressão emocional mais espontânea, afetando a qualidade dos relacionamentos. Além disso, como é comum nos testes projetivos, as variáveis devem ser interpretadas também na relação com outras variáveis do próprio teste, além de indicadores externos, de outros testes e do histórico da pessoa (Campos, 2013; Fensterseifer $\&$ Werlang, 2008). Nesse sentido, sugere-se que outras pesquisas continuem a verificar aspectos sintetizados sob o nome de inteligência emocional em outras variáveis dos instrumentos, como, por exemplo, o código EA-es do Zulliger, que diz respeito a adequada utilização de recursos e estratégias para lidar com estresse (Villemor-Amaral \& Primi, 2012) ou as frequências das cores no Pfister, que podem demonstrar diversos níveis da capacidade de utilização e controle das emoções. Mais especificamente como proposta de pesquisas futuras, a relação entre Formação em Camadas com Tapete com Início de Ordem poderia ser investigada, pois na presente amostra houve correlação significativa entre esses dois aspectos formais. Embora a correlação seja em magnitude mediana a baixa, o resultado sugere uma pequena sobreposição na avaliação, podendo haver a possibilidade de que os ambos meçam características psicológicas relacionadas.

Uma das limitações deste trabalho pode ser considerada o tamanho da amostra, provocada pela baixa frequência de respostas de cor, em geral. Seria importante que se conduzissem investigações visando obtenção de um número mínimo de respostas no Zulliger que garantisse melhor qualidade psicométrica de alguns resultados, tal como foi proposto pelo R-PAS (Meyer et al., 2011). Com isso, também se viabilizaria a ampliação da amostra, a fim de obter resultados mais generalizáveis.

É importante ainda destacar que, na amostra estudada, houve uma frequência incomum de utilização da cor Preta no Pfister, ao se comparar com as normas. Embora as cores não tenham sido utilizadas na análise de regressão, é possí- 
vel que sua frequência elevada esteja revelando aspectos psicológicos específicos da presente amostra. Além disso, o nível educacional dos participantes se concentrou em ensino médio completo e superior, havendo necessidade de ampliação para outras faixas educacionais.

\section{Referências}

Bash, K. W. (1984). Whole properties as determinants of determinants. Perceptual and Motor Skills, 59(3), 847-851. doi:10.2466/pms.1984.59.3.847

Berant, E., Mikulincer, M., Shaver, P. R., \& Segal, Y. (2005). Rorschach correlates of self-reported attachment dimensions: Dynamic manifestations of hyperactivating and deactivating strategies. Journal of Personality Assessment, 84(1), 7081. doi:10.1207/s15327752jpa8401_13

Bueno, J. M. H. (2008). Construção de um instrumento para avaliação da inteligência emocional em crianças (Tese de doutorado, Programa de Pós-Graduação Stricto Sensu em Psicologia, Universidade São Francisco, Itatiba, SP, Brasil). Recuperado em http://www.usf.edu.br/galeria/ getImage/427/6932606611633978.pdf

Bueno, J. M. H. (2013). Construção e validação de um instrumento para avaliação da regulação emocional. Estudos Interdisciplinares em Psicologia, 4(2), 186-200. doi:10.5433/2236$-6407.2013 \mathrm{v} 4 \mathrm{n} 2 \mathrm{p} 186$

Campos, R. C. (2013). Além dos números há uma pessoa: Sobre a utilização clínica de testes. Avaliação Psicológica, 12(3), 291-298.

Caruso, D. R., Salovey, P., Brackett, M., \& Mayer, J. D. (2015). The ability model of emotional intelligence. In S. Joseph (Ed.), Positive Psychology in Practice (pp. 543-558). Hoboken, NJ: John Wiley \& Sons.

Cohen, J. (1992). A power primer. Psychological Bulletin, 112(1), 155-159. doi:10.1037/00332909.112.1.155

Exner, J. E., Jr., \& Sendín, C. (1999). Manual de interpretação do Rorschach para o sistema compreensivo. São Paulo, SP: Casa do Psicólogo.

Fensterseifer, L., \& Werlang, B. S. G. (2008). Apontamentos sobre o status científico das técnicas projetivas. In A. E. Villemor-Amaral \& B. S. G. Werlang (Eds.), Atualizações em métodos projetivos para avaliação psicológica (pp. 15-33). São Paulo, SP: Casa do Psicólogo.
Hemphill, J. F. (2003). Interpreting the magnitudes of correlation coefficients. American Psychologist, 58(1), 78-79. doi:10.1037/0003-066X.58.1.78

Levy, J., \& Trevarthen, C. (1981). Color-matching, color-naming, and color-memory in split-brain patients. Neuropsychologia, 19, 523-541.

Malone, J. C., Stein, M. B., Slavin-Mulford, J., Bello, I., Sinclair, S. J., \& Blais, M. A. (2013). Seeing red: Affect modulation and chromatic color responses on the Rorschach. Bulletin of the Menninger Clinic, 77(1), 70-93. doi:10.1521/ bumc.2013.77.1.70

Mayer, J. D., Roberts, R. D., \& Barsade, S. G. (2008). Human abilities: Emotional intelligence. Annual Review of Psychology, 59, 507-536. doi:10.1146/ annurev.psych.59.103006.093646

Mayer, J. D., \& Salovey, P. (1999). O que é inteligência emocional? In P. Salovey \& D. J. Sluyter (Eds.), Inteligência emocional na criança: Aplicações na educação e no dia-a-dia (pp. 15-49). Rio de Janeiro, RJ: Campus.

Mayer, J. D., Salovey, P., \& Caruso, D. R. (2002). Mayer-Salovey-Caruso Emotional Intelligence Test (MSCEIT): User's manual. New York: Multi-Health Systems.

Meyer, G. J., \& Kurtz, J. E. (2006). Advancing personality assessment terminology: Time to retire "objective" and "projective" as personality test descriptors. Journal of Personality Assessment, 87(3), 223-225. doi:10.1207/ s15327752jpa8703_01

Meyer, G. J., Viglione, D. J., Mihura, J. L., Erard, R. E., \& Erdberg, P. (2011). Rorschach Performance Assessment System: Administration, coding, interpretation, and technical manual. Toledo, IL: Rorschach Performance Assessment System.

Miguel, F. K. (2010). O que sabemos sobre inteligência emocional. In G. Couto \& S. D. Pires (Eds.), Os contornos da psicologia contemporânea (pp. 73-104). São Paulo, SP: Casa do Psicólogo.

Miguel, F. K. (2014). Mitos e verdades no ensino de técnicas projetivas. Psico-USF, 19(1), 97-106. doi:10.1590/S1413-82712014000100010

O’Driscoll, C., Laing, J., \& Mason, O. (2014). Cognitive emotion regulation strategies, alexithymia and dissociation in schizophrenia, a review and meta-analysis. Clinical Psychology Review, 34(6), 482-495. doi:10.1016/j. cpr.2014.07.002 
Petrides, K. V., Pita, R., \& Kokkinaki, F. (2007). The location of trait emotional intelligence in personality factor space. British Journal of Psychology, 98(2), 273-289. doi:10.1348/000712606X120618

Strauss, G. P., Kappenman, E. S., Culbreth, A. J., Catalano, L. T., Lee, B. G., \& Gold, J. M. (2013). Emotion regulation abnormalities in schizophrenia: Cognitive change strategies fail to decrease the neural response to unpleasant stimuli. Schizophrenia Bulletin, 39(4), 872-883. doi:10.1093/schbul/sbs186

Villemor-Amaral, A. E. (2008). A validade teórica em avaliação psicológica. Psicologia: Ciência e Profissão, 28(1), 98-109. doi:10.1590/S141498932008000100008

Villemor-Amaral, A. E. (2012). As pirâmides coloridas de Pfister. São Paulo, SP: Casa do Psicólogo.

Villemor-Amaral, A. E., \& Primi, R. (2012). Teste de Zulliger no sistema compreensivo, ZSC: Forma individual. São Paulo, SP: Casa do Psicólogo.
Woyciekoski, C., \& Hutz, C. S. (2010). Inteligência emocional avaliada por autorrelato difere do construto personalidade? Psico-USF, 15(2), 151159. doi:10.1590/S1413-82712010000200003

Zeidner, M., Shani-Zinovich, I., Matthews, G., \& Roberts, R. D. (2005). Assessing emotional intelligence in gifted and non-gifted high school students: Outcomes depend on the measure. Intelligence, 33, 369-391. doi:10.1016/j.intell.2005.03.001

Recebido: 17/04/2016

$1^{a}$ revisão: 06/07/2016

$2^{a}$ revisão: $21 / 08 / 2016$

Aceite final: 30/09/2016 